\title{
Intratumoral Cellular Heterogeneity Critically Determines Extracellular Vesicle Uptake in Colorectal Cancer
}

\section{Andrea Kelemen}

Semmelweis University: Semmelweis Egyetem

Idan Carmi

Semmelweis University: Semmelweis Egyetem

\section{Adám Oszvald}

Semmelweis University: Semmelweis Egyetem

\section{Péter Lớrincz}

Eötvös Loránd Tudományegyetem: Eotvos Lorand Tudomanyegyetem

\section{Gábor Petővári}

Semmelweis University: Semmelweis Egyetem

\section{Tamás Tölgyes}

Uzsoki Hospital: Uzsoki Utcai Korhaz

\section{Kristóf Dede}

Uzsoki Hospital: Uzsoki Utcai Korhaz

\section{Attila Bursics}

Uzsoki Hospital: Uzsoki Utcai Korhaz

\section{Edit l. Buzás}

Semmelweis University: Semmelweis Egyetem

Zoltán Wiener ( $\nabla$ wiener.zoltan@med.semmelweis-univ.hu )

Semmelweis Egyetem https://orcid.org/0000-0001-7056-4926

\section{Research Article}

Keywords: IFITM1, exosome, patient-derived organoid, CRC

Posted Date: March 22nd, 2021

DOl: https://doi.org/10.21203/rs.3.rs-300126/v1

License: (c) (1) This work is licensed under a Creative Commons Attribution 4.0 International License.

Read Full License 


\section{Abstract}

The majority of colorectal cancer (CRC) patients carry mutations in the APC gene, which leads to an unregulated activation of the Wnt pathway. Extracellular vesicles (EV) are considered as potential therapeutic tools. Although CRC is a genetically heterogenous disease, the significance of the inter- and intra-tumor heterogeneity in EV uptake of CRC cells is not yet known. By using mouse and patient-derived organoids, the currently available best model of capturing cellular heterogeneity, we found that $A p C$ mutation induces the expression of interferon-induced transmembrane protein 1 (Ifitm1), a membrane protein that plays a major role in cellular antiviral responses. Importantly, organoids derived from IFITM $1^{\text {high }}$ CRC cells contained more proliferating cells. Whereas we found no difference in the uptake of liposomes between IFITM $1^{\text {high }}$ and IFITM $1^{\text {low/- }}$ cells, IFITM $1^{\text {high }}$ cells had a markedly reduced uptake of fibroblast EVs as compared to IFITM ${ }^{\text {low/- }}$ cells. In contrast, there was no difference in the intensity of EV release between CRC subpopulations with high and low IFITM1 level. Importantly, the difference in cell proliferation between these two subpopulations disappeared in the presence of fibroblast-derived EVs, proving the functional relevance of the enhanced EV uptake by IFITM1 low $C R C$ cells. Collectively, we identified CRC cell subpopulations with functional difference in their EV, but not liposome uptake ability that must be taken into consideration when using EVs as therapeutic tools for targeting cancer cells.

\section{Introduction}

Colorectal cancer (CRC) is a leading cause of cancer-related death in developed countries. In the vast majority of CRC patients, APC mutation is a central genetic event leading to the ligand-independent activation of the Wnt pathway and to the uncontrolled proliferation of adenoma cells. CRC is both genetically and phenotypically diverse, and other driver mutations of ten occur such as KRAS, TP53, inactivation of the TGF $\beta$ signalling pathway etc. This accumulation of mutations results in the progression of adenomas to invasive carcinomas. Recently, a common clustering of CRC has been suggested with CMS1-CMS4 subtypes that have characteristic mutation profiles [1]. However, CRC not only shows a large diversity among patients, but also an intra-tumoral cellular heterogeneity that is critical in therapy. Of note, one of the most state-of-the-art methods to capture this intra-tumoral heterogeneity of human cancers is the organoid technology [2,3]. Organoids are derived from patient samples and they are cultured in 3D matrices, such as Matrigel under well-defined conditions.

Interestingly, the activity of the Wnt pathway is influenced not only by mutations but also by other factors, such as hepatocyte growth factor (HGF) as well. As a consequence, Wnt signalling intensity is different among cells from the same tumor [4]. This leads to specific Wnt target gene expression patterns and to tumor cells with functional diversity. For example, the Wnt target LGR5 is induced only at a high Wnt activity. Although nowadays the identity and the function of CRC stem cells are intensively discussed, LGR5 expression is agreed to mark a CRC cell population with stem cell properties [5, 6].

The accumulation and activation of fibroblasts are hallmarks of some CRC subtypes. Importantly, there is a strong negative correlation between the amount of cancer-associated fibroblasts (CAF) and the time to 
disease relapse in CRC [7]. Furthermore, stromal gene expression signature predicts poor survival in patients [7]. CRC cells may undergo epithelial-mesenchymal transition and they may acquire a stromal expression pattern for a limited set of genes [8]. However, the predictive value of the stromal signature can at least partially be attributed to CAFs in the tumor tissues. Importantly, by secreting growth factors, such as HGF, CAFs contribute to the intra-tumoral cellular heterogeneity as well [4].

Extracellular vesicles (EV) are membrane-enclosed structures carrying biologically important molecules, such as proteins, lipids and RNAs between cells. Thus, they represent a special form of the intercellular communication. EVs can be classified according to their biogenesis, and they can be characterized by their biophysical or biochemical properties, too [9]. While exosomes are derived from the multivesicular bodies of the endosomal-lyosomal compartment, microvesicles are shed from the plasma membrane and they represent a larger EV population. In addition, large vesicles e.g. apoptotic bodies released by dying cells are regarded as EVs as well [10]. Since isolating EVs based on their cellular origin is difficult, they are often characterized by their size upon purification with differential centrifugation and ultracentrifugation [11]. Since EVs can be edited and loaded with specific molecules which are then transported in a protected way, furthermore, EVs are efficiently taken up by target cells, they hold a great promise for cancer therapies as well [12]. However, whether intra-tumoral cellular heterogeneity has a role in EV uptake and whether specific CRC subpopulations can be targeted by EVs more efficiently as compared to others, are not yet known.

Members of the interferon-induced transmembrane (IFITM) family regulate virus uptake in different compartments of the cells, thus, they critically contribute to cellular resistance against a wide range of membrane-surrounded viruses [13]. Among IFITM proteins, IFITM1 functions in the plasma membrane $[14,15]$. By using mouse intestinal and patient-derived organoids, here we provide evidence that differential IFITM1 expression marks an intra-tumoral cellular heterogeneity in CRC with a significantly different capacity for EV uptake. In addition, our results show that intra-tumoral cellular heterogeneity of CRC in IFITM1 expression has a major impact on fibroblast-derived EV function. Since the potential use of EVs in therapies is critically influenced by their uptake in tumor cells, our results may have an important consequence when designing EV-based targeting tools.

\section{Materials And Methods}

\section{Cell culture}

HT29 cells (ATCC HTB-38, American Type Culture Collection) and normal human colon fibroblasts (CCD18Co, ATCC-1459) were cultured in DMEM containing $4500 \mathrm{~g} / \mathrm{L}$ glucose (Gibco), 10\% FBS (Biosera), glutamine (Sigma) and 1x penicillin/streptomycin (Gibco). Cells were washed with phosphate buffered saline (PBS) three times and cultured in serum-free medium for 2 days. Medium was then changed and EVs were collected for 2 days. Cell number was counted in a Burker chamber. We only used cells with a low (<p9) passage numbers after receiving them from ATCC. Cell cultures were regularly tested for Mycoplasma contamination with Hoechst staining and they were negative in our studies. 


\section{Human adenoma and CRC organoid cultures}

The Medical Research Council of Hungary (ETT-TUKEB, No 51323-4/2015/EKU) approved the experiments with human samples and informed consent was obtained from the patients. CRC and adenoma patient-derived samples were processed according to previously published protocols [16]. Digested cell clusters were isolated by centrifugation at $650 \mathrm{~g}$ for $2 \mathrm{~min}$ and they were embedded into growth factor-reduced, phenol red-free Matrigel (Corning). In addition, we used the CRC organoid lines previously established in our research group [17]. Organoids were cultured in CRC medium containing basal medium (advanced DMEM/F12, 10mM HEPES (Sigma), penicillin/streptomycin, glutamine) and additionally B27 supplements (Gibco), $1 \mathrm{mM}$ N-Acetyl-Cysteine, $10 \mathrm{mM}$ Nicotinamide, $50 \mathrm{ng} / \mathrm{mL}$ EGF (Peprotech), 500 nM A83-01 (Sigma), 10 uM SB202190-Monohydrochloride (Sigma). Furthermore, Y27632 (Rho kinase inhibitor, Sigma) was added for 3 days after passaging to avoid anoikis. Organoids were removed from Matrigel in every 5-6 days mechanically, they were centrifuged at $650 \mathrm{~g}$ for 5 min and then digested with TrypLE (Thermo Fisher) for 5-10 min. CRC cell clusters were then washed with basal medium and embedded into Matrigel again in a 1:3 ratio. Clinical data of the patients and characterization of the organoids are in Table S1. Organoids \#1-3 have already been published [17].

\section{Normal human colon organoids}

Normal colon tissue samples were collected from patients undergoing CRC surgical operation with a distance of more than $3 \mathrm{~cm}$ from the tumors [16]. Human colonic crypts were isolated according to [16] with some modifications. Samples were cut into $<0.5 \mathrm{~cm}$ pieces, washed with PBS five times and incubated in PBS containing $2 \mathrm{mM}$ EDTA (Sigma) for $30 \mathrm{~min}$ at $4{ }^{\circ} \mathrm{C}$. The tubes had been pre-coated with $0.1 \%$ bovine serum albumin (BSA, Sigma) before the isolation procedure. Fractions were taken with PBS + $0.1 \%$ BSA into tubes containing $3 \mathrm{ml}$ advanced DMEM/F12 medium (Gibco). Fractions with crypts were embedded into Matrigel and cultured in human organoid medium composed of CRC medium, supplemented with $100 \mathrm{ng} / \mathrm{ml}$ human noggin (Peprotech), $1000 \mathrm{ng} / \mathrm{ml}$ human R-Spondin1 (R\&D Systems), $100 \mathrm{ng} / \mathrm{ml}$ murine Wnt3a (Peprotech) and $1 \mathrm{nM}$ gastrin (Sigma). Medium was changed every 3 days.

\section{Mouse intestinal organoid cultures}

The veterinary authority (Pest County Government Office, Hungary) approved the experiments with mice. Small intestinal crypts from C57BI/6J (Jackson Laboratory) were isolated according to previously published protocols [18]. Approximately 1,000 crypts were embedded into Matrigel (Corning, $20 \mathrm{ul} /$ well in 48-well plates) and cultured in small intestinal medium (SIM) contained advanced DMEM/F12 with N2 and B27 supplements (Gibco), 10 mM HEPES (Sigma), $1 \mu$ M N-Acetyl-Cysteine (Sigma), glutamine, penicillin/streptomycin, antibiotic/antimycotic mix (Gibco), $100 \mathrm{ng} / \mathrm{ml}$ noggin and $50 \mathrm{ng} / \mathrm{ml} \mathrm{EGF}$ (Peprotech), $500 \mathrm{ng} / \mathrm{ml}$ mouse R-Spondin1 (R\&D Systems, Bio-Techne). Organoids were removed from Matrigel in every 5-6 days, mechanically disrupted by pipetting, they were centrifuged at $650 \mathrm{~g}$ for $5 \mathrm{~min}$ and then embedded into Matrigel. 
To produce Apc-mutant organoids, we used a previously published sgRNA sequence (sgRNA4, [19]) and cloned it into the lentiCRISPR v2 plasmid (Addgene 52961). Apc-mutant organoids were produced according to [19] and [17] and they were selected by omitting R-Spondin1, EGF and noggin from the culture medium 3 days after transfection. Selected $A p c$-mutant organoids were used $>6$ days after removing the growth factors.

\section{EV isolation for functional experiments}

Serum-free medium from cultured cells was collected after 3 days. In some experiments, fibroblasts or HT29 cells were cultured with Vybrant ${ }^{T M}$ Cell-Labeling Solution DiL dye for 10 min (according to the Cell in Suspension protocol of the manufacturer), the cells were extensively washed with DMEM-Glutamax medium (Gibco) twice to remove the free dye. After the medium was changed to serum-free after 2 days and labelled EVs were isolated after 3 days, the supernatants were serially centrifuged at $300 \mathrm{~g}$ for 5 min and $2000 \mathrm{~g}$ for $20 \mathrm{~min}$ at $16^{\circ} \mathrm{C}$ to remove cells and cell fragments. Samples were then centrifuged at $12,500 \mathrm{~g}$ for $30 \mathrm{~min}$. The pellet was washed with PBS, centrifuged again and used as the medium EV fraction (mEV, 12.5K fraction). Small EVs (sEV, 100K fraction) were ultracentrifuged (UC) at $100,000 \mathrm{~g}$ for 70 min at $4{ }^{\circ} \mathrm{C}$, the EV-containing pellet was resuspended in PBS and UC again. The EV containing pellets were then resuspended in PBS and they were used for functional experiments.

\section{$2 D$ experiments with organoid cells}

2D experiments with organoid cells were carried out according to [20] with modifications. Briefly, 24-well plates (Eppendorf) or 8-well culture slides (Falcon) were pre-coated with Matrigel and basal medium mixture in case of human CRC organoids 1:20 ratio, in case of mouse organoids 1:10 ratio for 1 hour at $37^{\circ} \mathrm{C}$. The liquid was then discarded and plates were dried at room temperature. $10^{4}$ sorted CRC cells were plated into each well. The supernatant was changed to fresh medium after 1 day and the cells were treated with DiL labelled large EVs. The treated cells were fixed with 4\% paraformaldehyde (PFA) for 20 min the next day. Cells were then incubated with Phalloidin-iFluor 488 Reagent (Abcam) for 20 min. After covering samples with ProLong Diamond antifade mountant countaining DAPI (Thermo Fisher), images were taken with a Zeiss LSM800 or Leica TCS SP8 confocal microscopes. For mouse intestinal organoid 2D experiments, organoids were removed from Matrigel, they were centrifuged at $650 \mathrm{~g}$ for $5 \mathrm{~min}$ and digested with TrypLE (Gibco) until we received a single cell suspension (5-15 min). Cells were then washed with PBS and they were cultured in SIM medium with the Rho kinase inhibitor Y27632 in 24-well plates (Eppendorf). Cells were used for EV uptake experiments at $24 \mathrm{~h}$.

\section{Nanoparticle Tracking Analysis (NTA)}

Cells and organoids were cultured in FBS-free medium or in the chemically defined CRC medium, respectively. The supernatants were harvested after 2 days, they were serially centrifuged at $300 \mathrm{~g}$ for 5 min and at $2000 \mathrm{~g}$ for $20 \mathrm{~min}$. After centrifugation, $100 \mu \mathrm{L}$ supernatant was diluted to $1 \mathrm{~mL}$ in PBS and particle concentrations and size distribution were measured on a ZetaView Z-NTA instrument (Particle Metrix). For each measurement, the cell positions were scanned at $25^{\circ} \mathrm{C}$. The following camera settings 
were used: auto expose, gain: 28.8 , offset: 0 , shutter: 100 , sensitivity: 80 . The videos were analyzed with a minimum area of 5 , maximum area of 1000 and a minimum brightness of 20 by the ZetaView Analyze software 8.05.10. When the EV release from different experimental groups was compared, cells were cultured under the same conditions (medium volume, time, tissue culture dish format) and particle concentration data were then normalized to cell number.

\section{Functional studies with EVs}

$10^{8}$ small EVs in $10 \mu \mathrm{L}$ were added to each well with CRC organoids ( $200 \mu \mathrm{L}$ medium, $20 \mu \mathrm{L}$ Matrigel, 48well tissue culture plates, Eppendorf). In some experiments, $\mathrm{mEV}$ s were incubated with the organoids in Eppendorf tubes in a total volume of $10 \mu \mathrm{L}$ for 1 hour before embedding them into the 3D matrix. DiL labelled mEVs or labelled sEVs were added to $2 \mathrm{D}$ cell cultures $\left(10^{6}\right.$ cells in $500 \mu \mathrm{L}$ medium) and the EV uptake was analyzed by confocal microscopy or flow cytometry (see below). The pixel intensity distribution on confocal images was calculated using a custom Python script (available from the authors upon request). Images were segmented using the Cellpose algorithm [21] to determine the cell boundaries for each cell. Pixel values were then extracted within the cell boundaries from the red channel to characterize EV uptake intensity.

\section{Liposome production}

The production and characterization of liposomes have been previously described [17]. The liposomes had a mean diameter of $105 \mathrm{~nm}$. They were labelled with $2.5 \mu \mathrm{L}$ DiO fluorescent dye (Thermo Fisher) in $500 \mathrm{uL}$ PBS for $10 \mathrm{~min}$ at $37^{\circ} \mathrm{C}$. They were then ultracentrifuged at $100,000 \mathrm{~g}$ for $70 \mathrm{~min}$ at 4 degree, washed with PBS and ultracentrifuged again. Cells were treated with $10^{10}$ liposomes in $10 \mu \mathrm{L}$ PBS.

\section{Flow cytometry and cell sorting}

Organoids were removed from the 3D matrix, they were centrifuged at $650 \mathrm{~g}$ for $5 \mathrm{~min}$, washed with PBS, mechanically disrupted by vigorous pipetting and they were then digested with TrypLE (Gibco, Thermo Fisher) until the organoids were dissociated into single cells (5-10 min). They were taken up in FACS buffer (PBS, 1mM EDTA, 25mM HEPES, 1\% BSA). Cells were labelled with primary antibodies for $15 \mathrm{~min}$

and then with secondary antibodies for $15 \mathrm{~min}$ at room temperature. 10,000 events were measured with a FACSCalibur instrument (Beckton Dickinson) or cell subpopulations were sorted by a fluorescent cell sorter (Sony SH800S) into tubes. Sorted cells were then centrifuged at $650 \mathrm{~g}$ for 10 min at $4^{\circ} \mathrm{C}$ and 10,000-20,000 cells were embedded into $20 \mu \mathrm{L}$ Matrigel droplets. The same cell numbers were used among experimental groups within the same experiment.

\section{Semi-quantitative analysis of EVs by anti-CD63 or anti-CD81-coated beads}

Conditioned media from fibroblasts or CRC organoids were harvested after 2 days and they were centrifuged at $300 \mathrm{~g}$ for $5 \mathrm{~min}$ and $2000 \mathrm{~g}$ for $20 \mathrm{~min}$. EVs were then bound to beads coated with antiCD63 (Thermo Fisher, 10606D) or anti-CD81 (Thermo Fisher, 10616D). Before incubating with the EV- 
containing samples, beads had been blocked with $0.1 \%$ BSA (Sigma) for $20 \mathrm{~min}$. Six $\mu \mathrm{L}$ and $20 \mu \mathrm{L}$ of the anti-CD81 or anti-CD63-coated beads were added to $200 \mu \mathrm{L}$ supernatant, respectively. Beads were incubated overnight at $4{ }^{\circ} \mathrm{C}$, they were then washed with PBS three times and the EVs were labelled with FITC-anti-CD81 or PE-anti-CD63. The percentage of positive beads was detected by flow cytometry (FACSCalibur) and they were normalized to cell number.

\section{Immunocytochemistry}

Cells were fixed in 4\% PFA for 20 min, blocked and permeabilized in blocking buffer (PBS with $0.1 \%$ BSA, $5 \%$ FBS and $0.1 \%$ Triton X-100). Cells were then incubated with primary antibodies at $4{ }^{\circ} \mathrm{C}$ overnight and then in secondary antibodies in blocking buffer for $2 \mathrm{~h}$ at room temperature. After covering samples with ProLong Diamond antifade mountant containing DAPI (Thermo Fisher), images were taken with a Zeiss LSM800 or Leica TCS SP8 confocal microscope. In some experiments with the labelled medium-sized EV fraction, the blocking buffer contained $0.05 \%$ saponin (BD Biosciences) instead of Triton X-100. The antibodies used are listed in Table S2.

\section{Whole-mount immunostaining}

CRC organoids were cultured in 8-well chamber slides (BD Biosciences), fixed in 4\% PFA for 20 min and washed with PBS. Blocking and permeabilization were carried out in whole-mount blocking buffer (WBB, containing $5 \% \mathrm{FBS}, 0.2 \%$ BSA, $0.3 \%$ Triton $\mathrm{X}-100$ in PBS) for $30 \mathrm{~min}$. Samples were incubated with primary antibodies at $4{ }^{\circ} \mathrm{C}$ overnight in WBB. After washing in PBS, labelled secondary antibodies were added overnight at $4{ }^{\circ} \mathrm{C}$. The organoids were then mounted with ProLong Diamond antifade mountant countaining DAPI (Thermo Fisher) and analyzed with Zeiss LSM800 or Leica TCS SP8 confocal microscopes. Images were evaluated by the Image J software. The antibodies used are listed in Table $\mathbf{S} \mathbf{2}$.

\section{Protein concentration measurement and simple Western (WES) analysis}

Cells were cultured in serum free medium, EVs were pelleted with serial centrifugation/ultracentrifugation, they were washed with PBS and then resuspended in $19 \mu \mathrm{l}$ PBS and $1 \mu \mathrm{l}$ complete ${ }^{\mathrm{TM}}$ Protease Inhibitor Cocktail (Roche). For WES analysis from cells, they were taken up in $10 \mu$ RIPA lysis buffer. The protein concentrations of the supernatants were measured with the Micro BCA Protein Assay Kit (Thermo Scientific) and NanoDrop ND-1000 spectrophotometer (Thermo Scientific). $3 \mu$ of the lysates containing $0.5 \mu \mathrm{g}$ protein were applied to Simple Western analysis WES (ProteinSimple) following the manufacturer's instructions. SM-W004 (for analysis between 12-230 kDa), DM-TP01 total protein detection kit, DM-001 anti-rabbit detection kit, DM-002 anti-mouse detection kit and PS-ST02EZ-8 EZ Standard Pack 2 were used (ProteinSimple). The primary antibodies are listed in Table S2. The results were evaluated with the Compass for SW4.0.1 software (ProteinSimple).

\section{Transmission electron microscopy}


The EV-containing pellet after UC was washed with PBS and then resuspended in $10 \mu \mathrm{L}$ PBS. $5 \mu \mathrm{L}$ droplet was dried on a 300 mesh grid (Electron Microscopy Sciences, USA). EVs were fixed with $4 \%$

glutaraldehyde for 10 minutes and the grid was washed with water three times. Samples were treated with $2 \%$ phosphotungstic acid, they were dried at RT and imaged with a JEM-1011 transmission electron microscope (JEOL, Japan) equipped with a Morada digital camera (Olympus, Japan) using the iTEM software (Olympus, Japan).

\section{RNA isolation and mRNA measurements from cells}

Total RNA was isolated with the miRNEasy Micro Kit (Qiagen) according to the manufacturer's protocol in $15 \mu \mathrm{L}$ water. In some experiments, cells were directly fluorescence sorted into Qiazol (Qiagen). RNA concentration was determined with a NanoDrop instrument. Half $\mu \mathrm{g}$ RNA (in $20 \mu \mathrm{L}$ final volume) was reverse transcribed with the SensiFAST cDNA Synthesis Kit (Bioline) and quantitative PCR reactions were carried out using the SYBRGreen method with the SensiFAST SYBR Hi-ROX Kit (Bioline) on an ABI $7900 \mathrm{HT}$ Fast real-time PCR instrument (384-well format, $5 \mu \mathrm{L} /$ well volume). Results were evaluated with the following formula: relative expression level $=2^{-\Delta C t}$, where $\Delta \mathrm{Ct}=\mathrm{Ct}$ (gene of interest) $\mathrm{Ct}$ (housekeeping gene). The primers are in Table S3.

\section{Bioinformatical analysis}

Data in the expressional data sets GSE37926 and GSE83513 were analyzed by the GEO2R online tool. Statistical significance for determining expression difference between two groups for individual genes was determined by the default moderated t-statistics and Benjamini and Hochberg false discovery rate with a $\mathrm{p}<0.05$ threshold.

\section{Statistical analysis}

Student's paired or unpaired t-tests, ANOVA, Mann-Whitney U-test or Kruskal-Wallis with Dunn post hoc test were applied with ${ }^{*} p<0.05,{ }^{*} p<0.01$ and ${ }^{* \star *} p<0.005$ significance levels. Microsoft Excel, SPSS version 25 and GraphPad softwares were used for statistical evaluation. Mean+SD or median and 25 percentile values are shown with $n=3-5$ biological replicates, unless otherwise indicated.

\section{Results}

\section{Ifitm1 expression is increased after Apc mutation}

Previously we identified a set of genes that is activated by the loss of p53 and the activation of the Wnt pathway (the p53-suppressed invasiveness signature, PSIS) and which may account for the induction of invasiveness [22]. To find genes that may be involved in the malignant behavior of CRC cells and in EV uptake by CRC cells, we focused on IFITM1, a member of the PSIS set and the interferon-induced gene family with cell surface localization. Oncomine data analysis (TCGA colorectal data sets, www.oncomine.org) showed that IFITM1 is highly overexpressed in CRC samples compared to normal 
colon and rectum (Fig. S1a), suggesting that IFITM1 is regulated by the Wnt pathway. To test this hypothesis, we established organoid cultures from wild type (WT) mouse small intestine and introduced Apc-mutation to constantly activate the Wnt pathway. As expected, we observed highly elevated RNA levels of the known Wnt targets $L g r 5$, Axin2 and Myc (Fig. S1b). In addition, Apc mutation resulted in a pronounced increase in the expression of Prox1, an intestine-specific Wnt target gene [23] and we detected a more than 2,500-fold increase in the expression of Ifitm 1 (Fig. S1c).

To further study the regulation of IFITM1, we isolated organoids from normal colon (NCO) and from the tumor (CRCO) of CRC patients. In addition, we obtained samples from patients diagnosed with colon adenoma (AO) (Table S1). AOs and CRCOs were cultured without the Wnt-agonist R-Spondin1 and Wnt3a, thus, samples were selected for organoids carrying $A P C$ mutation and harbouring a constitutively active Wnt pathway. Interestingly, we detected higher RNA levels of not only AXIN2, LGR5 and MYC, but also of IFITM1 in both AOs and CRCOs as compared to NCOs (Fig. S1d).

LGR5 marks a stem cell population in CRC and in intestinal adenomas $[5,6,24]$. To decide whether Ifitm1 expression is specific for the Lgr5+ cells, we analyzed microarray data from sorted Lgr $5^{\text {high }}$ and Lgr $5^{\text {low }}$ mouse intestinal adenoma cells [24]. Whereas the RNA levels of Cd44 and Cd133 (two other genes often considered as CRC stem cell markers) were higher in the Lgr5 ${ }^{\text {high }}$ cell population, we found no difference in Ifitm1 (Fig. S1e). Furthermore, whereas the RNA level of CD133 was higher in LGR5 ${ }^{\text {high }}$ compared to LGR5 ${ }^{\text {low }}$ human CRC organoid cells in RNA expression datasets [6], we found no difference in IFITM1 expression between the two cell populations (Fig. S1f). Collectively, these data suggest that IFITM1 is under the regulation of the Wnt pathway, but it is not specific for the LGR5 ${ }^{\text {high }}$ CRC cells with stem cell features.

\section{CRC patient-derived organoids have a heterogeneous expression of IFITM1}

Although the membrane topology of IFITM1 has been unknown for a long time, recent publications suggested that the C-terminal part of the protein faces the extracellular space [14, 15]. In line with these studies, we detected a flow cytometric signal with an antibody produced against the N-terminal part of IFITM1 only if cells had been permeabilized before labelling (Fig. 1a-b). In contrast, the antibody against the C-terminal part labelled cells without permeabilization as well (Fig. 1a-b). To study both the intertumor and intra-tumor heterogeneity in IFITM1 expression, we used CRC patient-derived organoids. Interestingly, we could not detect a large variation in the RNA level of this gene among samples (Fig. 1c).

However, immunostaining proved the intra-organoid cellular heterogeneity in IFITM1 expression (Fig. 1d). Thus, IFITM1 expression is heterogeneous among CRC organoid cells.

\section{The IFITM1 ${ }^{\text {high }}$ CRC population contains more proliferating cells}

We next sorted IFITM $1^{\text {high }}$ and IFITM $1^{\text {low }}$ CRC organoid cells (Fig. 2a), checked the difference in the IFITM1 protein expression of the fluorescence sorted cells by flow cytometry and capillary-based immunoblotting (Fig. 2a-b) and subjected the samples to RNA analysis. Interestingly, whereas we found 
no difference in the RNAs of the Wnt target genes AXIN2, LGR5 or MYC, IFITM1 ${ }^{\text {high }}$ cells expressed the mesenchymal marker genes VIM and ZEB1 at a lower level (Fig. 2c). Furthermore, we observed a decreased RNA expression of $C D 133$, a gene that had been connected to stem-like cells in previous publications (Fig. 2c). Surprisingly, we detected no difference in the number of organoids initiated by the two sorted cell populations (Fig. 2d). However, the diameter of the organoids derived from IFITM1 1 high cells was significantly higher. They contained more KI67+ proliferating cells, but there was no difference in the percentage of active caspase3+ apoptotic cells (Fig. 2e-f and Fig. S2a). Parallel with these findings, we observed lower RNA levels of the differentiation markers MUC2 (Goblet cells) and ALPI (enterocytes) in IFITM 1 high cell-derived organoids (Fig. S2b), showing the shift between proliferating and other cell types. Thus, although classical stem-like cell markers are not highly expressed in IFITM1 ${ }^{\text {high }}$ cells, they produce organoids with a higher proliferation potential compared to the IFITM1 ${ }^{\text {low }}$ population.

\section{IFITM1 ${ }^{\text {high }}$ and IFITM1 Iow CRC cells do not differ in their intensity of EV release}

The IFITM1 cell surface protein is involved in inhibiting the uptake of some membrane-surrounded virus particles $[25,26]$, raising the possibility that this molecule regulates EV traffic as well. To study this question, we first determined the stability of IFITM1 expression in sorted cell populations. Organoidderived CRC cells were viable on Matrigel-coated plates (Fig. S2C) and we found no difference in cell surface IFITM1 expression between cells cultured either 2D or 3D (Fig. S2d). Importantly, sorted cells maintained their IFITM $1^{\text {high }}$ or IFITM1 ${ }^{\text {low }}$ expression patterns at day 7, determined by RT-qPCR (Fig. 3a). Furthermore, IFITM1 ${ }^{\text {high }}$ CRC cells showed a higher IFITM1 expression by immunocytochemistry even after 3 or 7 days in 2D and 3D cultures, respectively (Fig. 3b). Thus, the pattern of IFITM1 expression is maintained both in short-term 2D and 3D cultures.

Previously, we proved that small EVs (sEV, with a diameter of 50-100 nm) are preferentially released from $3 D$ cultures into the medium [17]. sEVs can be captured from the organoid supernatants by anti-CD63 or anti-CD81-coated beads that are two widely used sEV markers and the percentage of positive beads can be detected by flow cytometry $[17,27]$. We proved the presence of sEVs in CRC organoid-derived supernatants by antibody-coated beads, Nanoparticle Tracking Analysis (NTA), transmission electron microscopy and capillary-based immunoblot after ultracentrifugation (Fig. S3a-d). Importantly, calnexin that is not associated with sEVs [9], was detected only in cell lysates, but not in SEV preparations. In contrast, the sEV marker TSG101 was present in the ultracentrifuged pellet (Fig. S3c). We next compared the SEV release from IFITM $1^{\text {high }}$ and IFITM1 ${ }^{\text {low }}$ cell-derived organoids. Unexpectedly, we found no difference in the SEV concentration between the two sorted cell population-derived organoids either with the bead-based semi-quantitative method (Fig. 3c-d) or with NTA (Fig. 3e-f). We obtained similar data when sorted cells were cultured in another matrix, collagen I or in a mixture of collagen I and Matrigel (Fig. S4a-d). These results suggest that IFITM1 $1^{\text {high }}$ and IFITM1 ${ }^{\text {low }}$ CRC cells do not have an altered release of sEVs. 
Next we studied whether cell populations with different Ifitm1 expression have different EV uptake abilities. To address this question, we cultured cells from WT and $A p c$ mutant mouse intestinal organoids in $2 \mathrm{D}$ conditions for short term and we added EVs isolated by centrifugation at $12,500 \mathrm{~g}$ (medium EV fraction, $\mathrm{mEV}$ ) from fibroblasts that had been pre-treated with a membrane labelling dye (Fig. S5a). Importantly, we found no increase in the percentage of active caspase+ cells after labelling, showing that this treatment does not induce apoptosis in fibroblasts (Fig. S5b). Since EVs were not directly labelled and cells were washed after applying the dye, this precluded the possibility that we measured dye aggregates. Importantly, we detected EVs in fibroblast culture supernatant by NTA measurements when isolating $\mathrm{mEV}$ s or sEVs (Fig. S5c). Furthermore, transmission electron microscopy proved the identity of EVs in samples after ultracentrifugation (Fig. S5d). In addition, capillary based-immunoblot showed the presence of the sEV marker TSG101 only in SEV preparations, whereas we could detect calnexin in the mEV fraction (Fig. S5e) [28].

Interestingly, Apc mutant organoids that express a higher level of Ifitm 1 accumulated less fibroblastderived mEVs compared to wild type intestinal organoids (Fig. S5f). To study whether EV uptake intensity differs between CRC cell subpopulations as well, we next sorted IFITM1 $1^{\text {high }}$ and IFITM1 ${ }^{\text {low }}$ cells and we cultured them with labelled mEVs or sEVs. Of note, less IFITM1 ${ }^{\text {high }}$ cells took up EVs both in case of HT29 CRC cell-line-derived and human colon fibroblast-derived $\mathrm{mEV}$ s as compared to IFITM ${ }^{\text {low }}$ CRC cells, detected by confocal microscopy (Fig. 4a-b). In addition, IFITM1 ${ }^{\text {high }}$ cells that had taken up fibroblastderived EVs showed a lower signal intensity for mEVs than IFITM ${ }^{\text {low }}$ cells (Fig. 4c). Thus, these results suggest that the difference in EV uptake is not restricted to one specific cell type-derived EVs. Importantly, we proved the presence of $\mathrm{mEVs}$ and sEVs in the conditioned medium of HT29 cells with NTA and transmission electron microscopy (Fig. S5g-h). In addition, when samples were treated with Triton X-100, the EV signal disappeared from the cells (Fig. 4d), proving that we visualized membrane-enclosed EVs in our experiments.

As the next experiment, we treated CRC organoid cells with fibroblast-derived labelled EVs in 2D cultures and then sorted cells with the highest and lowest fluorescent signal, representing cell populations with high and low EV uptake ability, respectively (Fig. 4e). Whereas we found no difference in the expression of AXIN2 and MYC, we measured a significantly higher RNA level of IFITM1 in cells with low EV uptake (Fig. 4f). Since we found no increase in the expression of IFITM1 in CRC cells after treatment with EVs (Fig. $\mathbf{4 g}$ ), this confirms again that IFITM $1^{\text {high }}$ cells take up less EVs. In addition, the majority of cells with high IFITM1 level were negative for the fluorescent signal after treatment with labelled fibroblast-derived mEVs (Fig. 4h). Furthermore, we observed the accumulation of IFITM1 $1^{\text {low }}$ cells within the $\mathrm{mEV}+$ cell population, measured by flow cytometry (Fig. 4i). When focusing on cells that had taken up mEVs, we detected a higher signal intensity, characterizing a higher mEV uptake, within the IFITM1 ${ }^{\text {low }}$ cell population (Fig. 4j). Importantly, we obtained similar results when CRC organoid cells were treated with the sEV fraction collected from labelled fibroblasts or HT29 cells (Fig. 4i-j). Collectively, all these data prove i) the presence of cell subclones in CRC with different $\mathrm{mEV}$ and $\mathrm{sEV}$ uptake ability and ii) that IFITM1 ${ }^{\text {high }}$ marks cells with a lower EV uptake. 
To study whether this difference in uptake is specific for EVs, we produced fluorescently labelled synthetic liposomes and we added them to sorted IFITM $1^{\text {high }}$ and IFITM $1^{\text {low }}$ CRC cells after ultracentrifugation. In contrast to the labelled or unlabelled EV preparates, we detected no signal when only the fluorescent dye was ultracentrifuged (Fig. S5i), thus, ruling out the possibility that we used dye aggregates in our experiments. Surprisingly, we found no difference in the signal intensity between the two cell populations after adding fluorescently labelled synthetic liposomes (Fig. 4k-I). Thus, the difference in EV uptake ability between the two CRC cell subpopulations is specific for the EVs.

\section{Fibroblast-derived EVs result in a marked increase in the proliferating cell number of IFITM1'ow CRC organoids}

To test the functional relevance of differential EV uptake between CRC cell subpopulations, we first added fibroblast-derived mEVs or sEVs to CRC cells and we then detected IFITM1 expression and KI67 in the organoids after 7 days. Importantly, neither sEVs nor mEVs resulted in a change in the percentage of IFITM1+ CRC cells (Fig. 5a). However, we detected a markedly higher increase in the proportion of proliferating cells within the IFITM1- cell population than in IFITM1+ cells in the presence of EVs (Fig. 5b). Furthermore, adding fibroblast-derived $\mathrm{mEVs}$ or $\mathrm{sEV}$ s to sorted IFITM1 ${ }^{\text {low }}$ cells resulted in organoids with a markedly higher increase in the number of KI67+ cells as compared to IFITM1 ${ }^{\text {high }}$ cell-derived organoids (Fig. 5c). In addition, the initial difference in the percentage of proliferating cells between the two CRC cell subpopulations completely disappeared after treatment with mEVs or sEVs (Fig. 5c). On the other hand, we detected no changes in the percentage of active caspase-3+ apoptotic cells (Fig. 5d). Thus, these results indicate that differential $\mathrm{mEV}$ or $\mathrm{sEV}$ uptake by $\mathrm{CRC}$ cell subpopulations critically modifies the percentage of proliferating cells in an organoid model.

\section{Discussion}

EVs can be edited and loaded with specific molecules. Although EVs are considered as a promising tool for cancer therapy, it is still unknown which CRC cell populations take up EVs preferentially. By using patient-derived organoids, here we identify CRC subpopulations with different EV uptake capability. Importantly, whereas CRC cells expressing the cell surface protein IFITM1 at a high level (IFITM1 ${ }^{\text {high }}$ ) and at a low level (IFITM1 ${ }^{\text {low }}$ ) do not differ in their EV release intensity, IFITM1 ${ }^{\text {high }}$ cells take up significantly less EVs. Fibroblast-derived EVs result in a marked increase in the proliferating cell number of only IFITM $1^{\text {low }} \mathrm{CRC}$ organoids, showing the functional significance of the difference in EV uptake. We also show that Ifitm 1 expression is increased after $A p c$ mutation, similarly to human adenomas when compared to normal colonic epithelium. CRC patient-derived organoids have a heterogenous intraorganoid expression of IFITM1 and the IFITM 1 high CRC population contains more proliferating cells.

IFITM family members have been identified as molecular markers in human colorectal tumors [29]. Other published studies have also connected IFITM1 to the progression of colorectal cancer, showing that it modifies the proliferating, invasive and metastatic capability of CRC cell lines [30]. However, the majority of these previous data were derived from models using cell lines cultured 2D. Importantly, our study is the 
first where patient-derived organoids were used to characterize IFITM $1^{+} \mathrm{CRC}$ cells. We identified the existence of CRC intra-tumor cellular heterogeneity in IFITM1 expression and we observed that IFITM1 1 high sorted cells form organoids with more proliferating cells, suggesting that IFITM1 $1^{\text {high }}$ and IFITM1 ${ }^{\text {low }}$ subpopulations differentially contribute to CRC tumorigenesis.

IFITM genes are generally regulated by type I and type II interferons. Interestingly however, IFITM3 is constitutively expressed in tissue-resident $T$ cells in some tissues, such as the airways and the lungs [31]. In T cells, IFITM2 and IFITM3 expressions are regulated by T-cell receptor (TCR) signal transduction [32], and hedgehog $(\mathrm{Hh})$ signalling modifies the expression of IFITM genes in some systems [33]. This shows diverse regulatory mechanisms for this gene cluster. Here we prove that human organoids derived from adenomas or CRC patients express IFITM1 at a higher level compared to normal colon. By using genetically modified mouse intestinal organoids, we provide evidence that $A p c$ mutation is a critical factor of the regulation of Ifitm 1 expression. Our findings concur with those of Lickert $\mathrm{H}$ et al [34] who found a regulatory effect of the Wnt pathway on Ifitm genes during gastrulation. In addition, we confirmed the results by Andreu $\mathrm{P}$ et al [35], showing the regulation of many Ifitm members in mouse intestinal adenoma models.

The IFITM genes encode for a group of small homologous proteins that are localized in the membranes of the endosomal-lysosomal compartment or the plasma membrane [36]. While there are seven Ifitm genes in mice, only five of them have been identified in the human genom. However, IFITM1 is among the genes shared by both species. IFITM proteins provide a cellular resistance against a wide variety of both enveloped and non-enveloped viruses not only in vitro, but in vivo as well [37, 38]. Similarly to certain viruses, EVs contain a membrane envelope. Although the mechanism of action of the IFITM proteins is largely unknown, some studies suggested that they exert their anti-viral effects via changing the fluidity or other physical properties of cell membranes [39,40] which may apply for EV uptake as well. Of note, here we show that IFITM1 ${ }^{\text {high }}$ cells take up less EVs compared to cells with low IFITM1 expression, however, this difference was not characteristic for synthetic liposomes. Thus, our results clearly indicate the existance of functional heterogeneity in EV, but not liposome uptake within CRC. In addition, these data provide further evidence for the relationship between certain viruses and EVs not only in the mechanism of their production, but in their uptake as well [41]. The biological importance of this differential EV uptake is illustrated by our data that fibroblast-derived EVs have a more pronounced stimulatory effect on cell proliferation in the IFITM1 ${ }^{\text {low }}$ CRC subpopulation that accumulate more EVs.

Unlike liposomes and other synthetic drug nanoparticle carriers, EVs contain proteins in their membranes that enhance their uptake, thus, providing a more powerful delivery system of drugs or RNAs in RNA interference (RNAi)-based approaches. The usability of this approach has recently been proved by two elegant studies where RNAi was delivered to specifically target oncogenic KRas in pancreatic tumors in mouse models [42, 43]. Importantly, these results open the possibility for engineered EV-based therapies targeting oncogenes in other cancers, such as CRC. However, the proper and efficient use of EVs delivering the engineered cargo critically depends on their uptake by tumor cell subpopulations. As an 
important step on this way, we provide evidence for the intra-tumor cellular heterogeneity of EV uptake intensity in CRC. Interestingly, the IFITM 1 high cell population with a higher proliferation potential accumulates less EVs, a fact that must be considered when designing targeting EVs for clinical applications in CRC.

\section{Declarations}

Funding: This study was financed by OTKA-NN 118018, the National Competitiveness and Excellence Program NVKP_16-0007 and NVKP_16-0017 (all provided by the National Research, Development and Innovation Office, Hungary), by the Higher Education Institutional Excellence Programme (Ministry for Innovation and Technology, Hungary) within the framework of the Molecular Biology and Therapeutic thematic programmes of the Semmelweis University, by the Semmelweis Scientific and Innovation Fund (Hungary), by VEKOP-2.3.3-15-2016-00007 and VEKOP-2.3.3-15-2017-00016 (Hungary). Andrea Kelemen, Péter Lőrincz and Zoltán Wiener are supported by the Richter Gedeon Foundation, PPD-222/2018 (Premium Postdoctoral Research Program, Hungarian Academy of Sciences) and János Bolyai Research Fellowship (BO/00131/20/8, Hungarian Academy of Sciences), respectively. Zoltán Wiener receives a grant from the Ministry of Innovation and Technology, Hungary (ÚNKP New National Excellence Program, ÚNKP-20-5-SE-18).

Conflict of interest: The authors declare that they have no conflict of interest.

Ethics approval : The Medical Research Council of Hungary (ETT-TUKEB, No 51323-4/2015/EKU) as the national authority approved the experiments with human samples and informed consent was obtained from the patients. Mouse experiments were carried out according to and with the approval of the Institutional Animal Care and Use Committee (Semmelweis University). The Pest County Government Office of Hungary (the competent veterinary authority) approved the maintenance and experiments with mice. Animal experiments followed the principles of the Declaration of Helsinki.

Consent to participate and publication : All participants approved the submission and publication of the manuscript. Informed consent was obtained from all individual participants included in the study.

Availability of data and material : Data are available from the corresponding author upon reasonable request.

Code availability : N/A

\section{Authors' contributions :}

AK: Conception and design, collection and/or assembly of data, data analysis and interpretation, manuscript writing

IC, ÁO, PL, GP : Data collection 
TT, KD, AB: Provision of study material or patients

ElB: Data interpretation, writing the manuscript

ZW: Conception and design, financial support, data analysis and interpretation, manuscript writing, final approval of manuscript

Acknowledgments: We thank to all members of the Extracellular Vesicle Research Group and to the Molecular Cancer Biology Research Group (Semmelweis University, Department of Genetics, Cell and Immunobiology) for their help. We acknowledge Dr. Zoltán Varga (Research Centre for Natural Sciences, Institute of Materials and Environmental Chemistry, Eötvös Loránd Research Network, Hungary) for providing liposomes.

\section{References}

1. Guinney J, Dienstmann R, Wang X, de Reynies A, Schlicker A, Soneson C, Marisa L et al (2015) The consensus molecular subtypes of colorectal cancer. Nat Med 21:1350-6.

2. Ganesh K, Wu C, O'Rourke KP, Szeglin BC, Zheng Y, Sauve CG, Adileh M et al (2019) A rectal cancer organoid platform to study individual responses to chemoradiation. Nat Med 25:1607-1614.

3. de Witte CJ, Espejo Valle-Inclan J, Hami N, Lohmussaar K, Kopper O, Vreuls CPH, Jonges GN et al (2020) Patient-Derived Ovarian Cancer Organoids Mimic Clinical Response and Exhibit Heterogeneous Inter- and Intrapatient Drug Responses. Cell Rep 31:107762.

4. Vermeulen L, De Sousa EMF, van der Heijden M, Cameron K, de Jong JH, Borovski T, Tuynman JB et al (2010) Wnt activity defines colon cancer stem cells and is regulated by the microenvironment. Nat Cell Biol 12:468-76.

5. Cortina C, Turon G, Stork D, Hernando-Momblona X, Sevillano M, Aguilera M, Tosi S et al (2017) A genome editing approach to study cancer stem cells in human tumors. EMBO Mol Med 9:869-879.

6. Shimokawa M, Ohta Y, Nishikori S, Matano M, Takano A, Fujii M, Date S et al (2017) Visualization and targeting of LGR5+ human colon cancer stem cells. Nature 545:187-192.

7. Calon A, Lonardo E, Berenguer-Llergo A, Espinet E, Hernando-Momblona X, Iglesias M, Sevillano M et al (2015) Stromal gene expression defines poor-prognosis subtypes in colorectal cancer. Nat Genet.

8. Vellinga TT, den Uil S, Rinkes IH, Marvin D, Ponsioen B, Alvarez-Varela A, Fatrai S et al (2016) Collagen-rich stroma in aggressive colon tumors induces mesenchymal gene expression and tumor cell invasion. Oncogene 35:5263-5271.

9. Thery C, Witwer KW, Aikawa E, Alcaraz MJ, Anderson JD, Andriantsitohaina R, Antoniou A et al (2018) Minimal information for studies of extracellular vesicles 2018 (MISEV2018): a position statement of the International Society for Extracellular Vesicles and update of the MISEV2014 guidelines. $J$ Extracell Vesicles 7:1535750.

10. Mathieu M, Martin-Jaular L, Lavieu G, Thery C (2019) Specificities of secretion and uptake of exosomes and other extracellular vesicles for cell-to-cell communication. Nat Cell Biol 21:9-17. 
11. Kowal J, Arras G, Colombo M, Jouve M, Morath JP, Primdal-Bengtson B, Dingli F et al (2016) Proteomic comparison defines novel markers to characterize heterogeneous populations of extracellular vesicle subtypes. Proc Natl Acad Sci U S A 113:E968-77.

12. Esmaeili A, Hosseini S, Baghaban Eslaminejad M (2021) Engineered-extracellular vesicles as an optimistic tool for microRNA delivery for osteoarthritis treatment. Cell Mol Life Sci 78:79-91.

13. Rojas JM, Alejo A, Martin V, Sevilla N (2021) Viral pathogen-induced mechanisms to antagonize mammalian interferon (IFN) signaling pathway. Cell Mol Life Sci 78:1423-1444.

14. Smith SE, Busse DC, Binter S, Weston S, Diaz Soria C, Laksono BM, Clare S et al (2019) InterferonInduced Transmembrane Protein 1 Restricts Replication of Viruses That Enter Cells via the Plasma Membrane. J Virol 93.

15. Weston S, Czieso S, White IJ, Smith SE, Kellam P, Marsh M (2014) A membrane topology model for human interferon inducible transmembrane protein 1. PLoS One 9:e104341.

16. Sato T, Stange DE, Ferrante M, Vries RG, Van Es JH, Van den Brink S, Van Houdt WJ et al (2011) Long-term expansion of epithelial organoids from human colon, adenoma, adenocarcinoma, and Barrett's epithelium. Gastroenterology 141:1762-72.

17. Szvicsek Z, Oszvald A, Szabo L, Sandor GO, Kelemen A, Soos AA, Paloczi K et al (2019) Extracellular vesicle release from intestinal organoids is modulated by Apc mutation and other colorectal cancer progression factors. Cell Mol Life Sci 76:2463-2476.

18. Sato T, Vries RG, Snippert HJ, van de Wetering M, Barker N, Stange DE, van Es JH et al (2009) Single Lgr5 stem cells build crypt-villus structures in vitro without a mesenchymal niche. Nature 459:262-5.

19. Schwank G, Koo BK, Sasselli V, Dekkers JF, Heo I, Demircan T, Sasaki N et al (2013) Functional repair of CFTR by CRISPR/Cas9 in intestinal stem cell organoids of cystic fibrosis patients. Cell Stem Cell 13:653-8.

20. Thorne CA, Chen IW, Sanman LE, Cobb MH, Wu LF, Altschuler SJ (2018) Enteroid Monolayers Reveal an Autonomous WNT and BMP Circuit Controlling Intestinal Epithelial Growth and Organization. Dev Cell 44:624-633 e4.

21. Stringer C, Wang T, Michaelos M, Pachitariu M (2021) Cellpose: a generalist algorithm for cellular segmentation. Nat Methods 18:100-106.

22. Elyada E, Pribluda A, Goldstein RE, Morgenstern Y, Brachya G, Cojocaru G, Snir-Alkalay I et al (2011) CKlalpha ablation highlights a critical role for p53 in invasiveness control. Nature 470:409-13.

23. Petrova TV, Nykanen A, Norrmen C, Ivanov KI, Andersson LC, Haglund C, Puolakkainen P et al (2008) Transcription factor PROX1 induces colon cancer progression by promoting the transition from benign to highly dysplastic phenotype. Cancer Cell 13:407-19.

24. Schepers AG, Snippert HJ, Stange DE, van den Born M, van Es JH, van de Wetering M, Clevers $H$ (2012) Lineage tracing reveals Lgr5+ stem cell activity in mouse intestinal adenomas. Science 337:730-5.

25. Smith S, Weston S, Kellam P, Marsh M (2014) IFITM proteins-cellular inhibitors of viral entry. Curr Opin Virol 4:71-7. 
26. Bailey CC, Zhong G, Huang IC, Farzan M (2014) IFITM-Family Proteins: The Cell's First Line of Antiviral Defense. Annu Rev Virol 1:261-283.

27. Oszvald A, Szvicsek, Z., Pápai, M., Kelemen, A., Varga, Z., Tölgyes, T., Dede, K., Bursics, A., Buzás, E.I., Wiener, Z. (2020) Fibroblast-Derived Extracellular Vesicles Induce Colorectal Cancer Progression by Transmitting Amphiregulin. Front Cell Dev Biol.

28. Haraszti RA, Didiot MC, Sapp E, Leszyk J, Shaffer SA, Rockwell HE, Gao F et al (2016) High-resolution proteomic and lipidomic analysis of exosomes and microvesicles from different cell sources. $J$ Extracell Vesicles 5:32570.

29. Liang R, Li X, Zhu X (2020) Deciphering the Roles of IFITM1 in Tumors. Mol Diagn Ther 24:433-441.

30. Sari IN, Yang YG, Phi LT, Kim H, Baek MJ, Jeong D, Kwon HY (2016) Interferon-induced transmembrane protein 1 (IFITM1) is required for the progression of colorectal cancer. Oncotarget 7:86039-86050.

31. Wakim LM, Gupta N, Mintern JD, Villadangos JA (2013) Enhanced survival of lung tissue-resident memory CD8(+) T cells during infection with influenza virus due to selective expression of IFITM3. Nat Immunol 14:238-45.

32. Yanez DC, Sahni H, Ross S, Solanki A, Lau Cl, Papaioannou E, Barbarulo A et al (2019) IFITM proteins drive type $2 \mathrm{~T}$ helper cell differentiation and exacerbate allergic airway inflammation. Eur $\mathrm{J}$ Immunol 49:66-78.

33. Furmanski AL, Barbarulo A, Solanki A, Lau Cl, Sahni H, Saldana JI, D'Acquisto F et al (2015) The transcriptional activator Gli2 modulates T-cell receptor signalling through attenuation of AP-1 and NFkappaB activity. J Cell Sci 128:2085-95.

34. Lickert H, Cox B, Wehrle C, Taketo MM, Kemler R, Rossant J (2005) Dissecting Wnt/beta-catenin signaling during gastrulation using RNA interference in mouse embryos. Development 132:2599-609.

35. Andreu P, Colnot S, Godard C, Laurent-Puig P, Lamarque D, Kahn A, Perret C et al (2006) Identification of the IFITM family as a new molecular marker in human colorectal tumors. Cancer Res 66:1949-55.

36. Liao Y, Goraya MU, Yuan X, Zhang B, Chiu SH, Chen JL (2019) Functional Involvement of InterferonInducible Transmembrane Proteins in Antiviral Immunity. Front Microbiol 10:1097.

37. Savidis G, Perreira JM, Portmann JM, Meraner P, Guo Z, Green S, Brass AL (2016) The IFITMs Inhibit Zika Virus Replication. Cell Rep 15:2323-30.

38. Everitt AR, Clare S, Pertel T, John SP, Wash RS, Smith SE, Chin CR et al (2012) IFITM3 restricts the morbidity and mortality associated with influenza. Nature 484:519-23.

39. Chesarino NM, Compton AA, McMichael TM, Kenney AD, Zhang L, Soewarna V, Davis M et al (2017) IFITM3 requires an amphipathic helix for antiviral activity. EMBO Rep 18:1740-1751.

40. Li K, Markosyan RM, Zheng YM, Golfetto O, Bungart B, Li M, Ding S et al (2013) IFITM proteins restrict viral membrane hemifusion. PLoS Pathog 9:e1003124.

41. Nolte-'t Hoen E, Cremer T, Gallo RC, Margolis LB (2016) Extracellular vesicles and viruses: Are they close relatives? Proc Natl Acad Sci U S A 113:9155-61. 
42. Mendt M, Kamerkar S, Sugimoto H, McAndrews KM, Wu CC, Gagea M, Yang S et al (2018) Generation and testing of clinical-grade exosomes for pancreatic cancer. JCI Insight 3.

43. Kamerkar S, LeBleu VS, Sugimoto H, Yang S, Ruivo CF, Melo SA, Lee JJ et al (2017) Exosomes facilitate therapeutic targeting of oncogenic KRAS in pancreatic cancer. Nature 546:498-503.

\section{Figures}

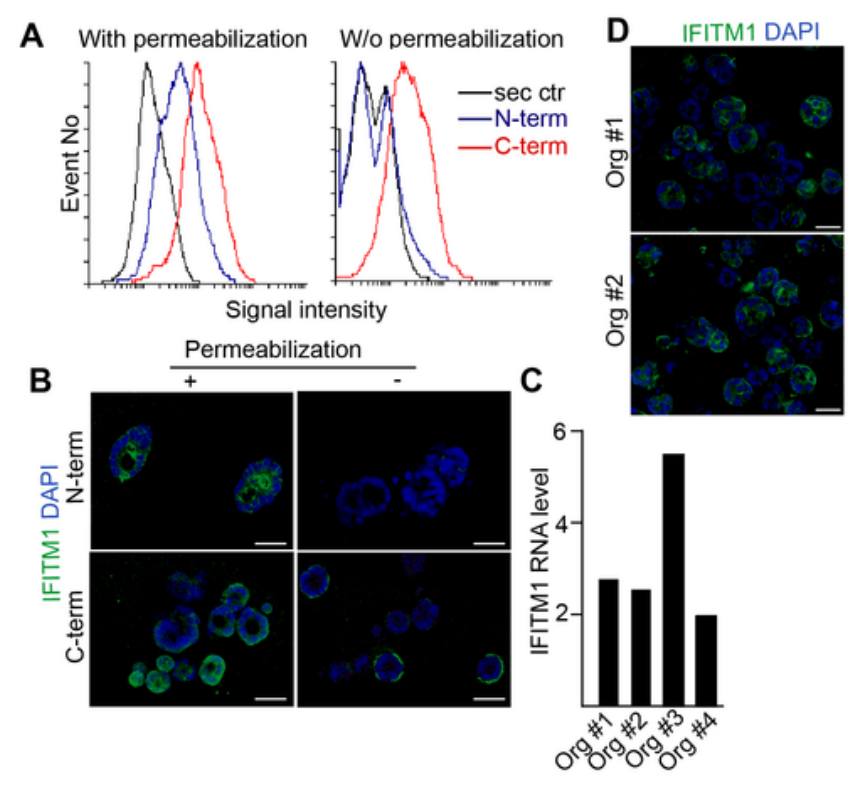

Kelemen, Figure 1 


\section{Figure 1}

Expression heterogeneity of IFITM1 in human CRC organoids. A) Flow cytometry with antibodies specific for the N-terminal (N-term) or C-terminal (C-term) parts of IFITM1. Note that samples were either directly labelled or they were fixed and permeabilized before labelling. B) Whole-mount immunocytochemistry of CRC organoids for IFITM1 with antibodies binding to the N-terminal or C-terminal parts of the protein. Immunostaining was carried out with/without permeabilization (org \#1). C) IFITM1 RNA levels compared to HPRT1 housekeeping from four CRC organoid lines (RT-qPCR). D) IFITM1 expression in two CRC organoid lines with the antibody recognizing the $\mathrm{C}$-terminal part of the protein (whole-mount immunostaining). Scale bars: $50 \mu \mathrm{m}(\mathrm{B}, \mathrm{D})$. 

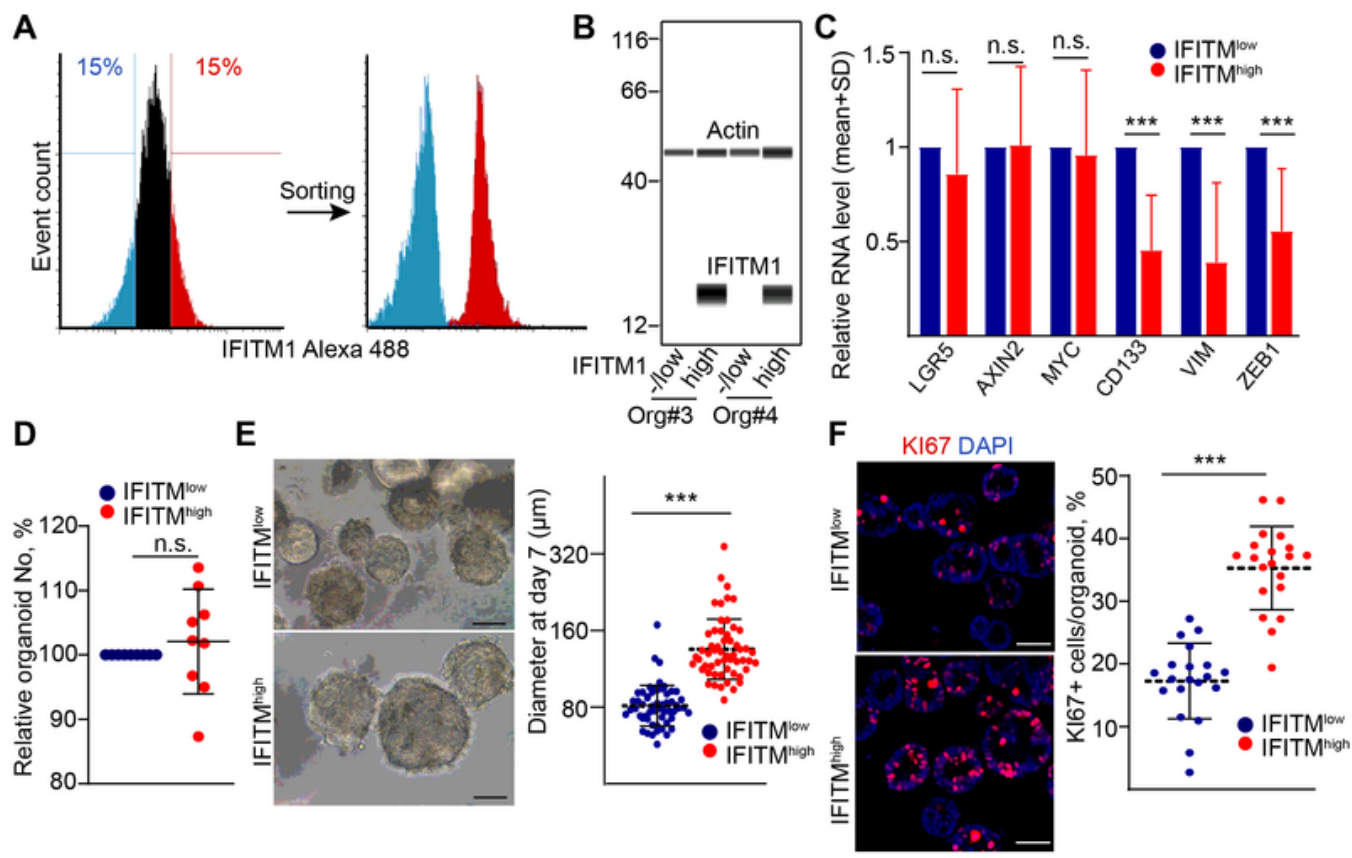

Kelemen, Figure 2.

\section{Figure 2}

The IFITM1 high CRC cell-derived organoids contain more proliferating cells than IFITM1 low organoids. A) The fluorescence sorting strategy for IFITM1 high and IFITM1 low cells and their analysis after sorting with flow cytometry. B) IFITM1 protein levels of cells sorted from two CRC organoid lines (capillary-based immunoblotting). Note that actin was used as housekeeping control. C) Relative RNA levels of the indicated genes in sorted cells (RT-qPCR, n=4). D) The relative number of organoids initiated by 20,000 
sorted cells. Each dot represents an individual sorting experiment. E) Representative images (left panel) and the diameter of CRC organoids (right panel) derived from IFITM1low or IFITM1high sorted cells. F) The percentage of KI67+ proliferating cells. Representative images and their quantification from confocal images (whole mount immunostaining). For $E$ ) and $F$ ), quantifications were carried out from ten images taken from four sorting experiments. Scale bars: $50 \mu \mathrm{m}$ (E, F). Paired t-test (C, D) and Mann-Whitney Utest $(E, F)$ were used. $* * *<0.005$, n.s.: $p>0.05$.

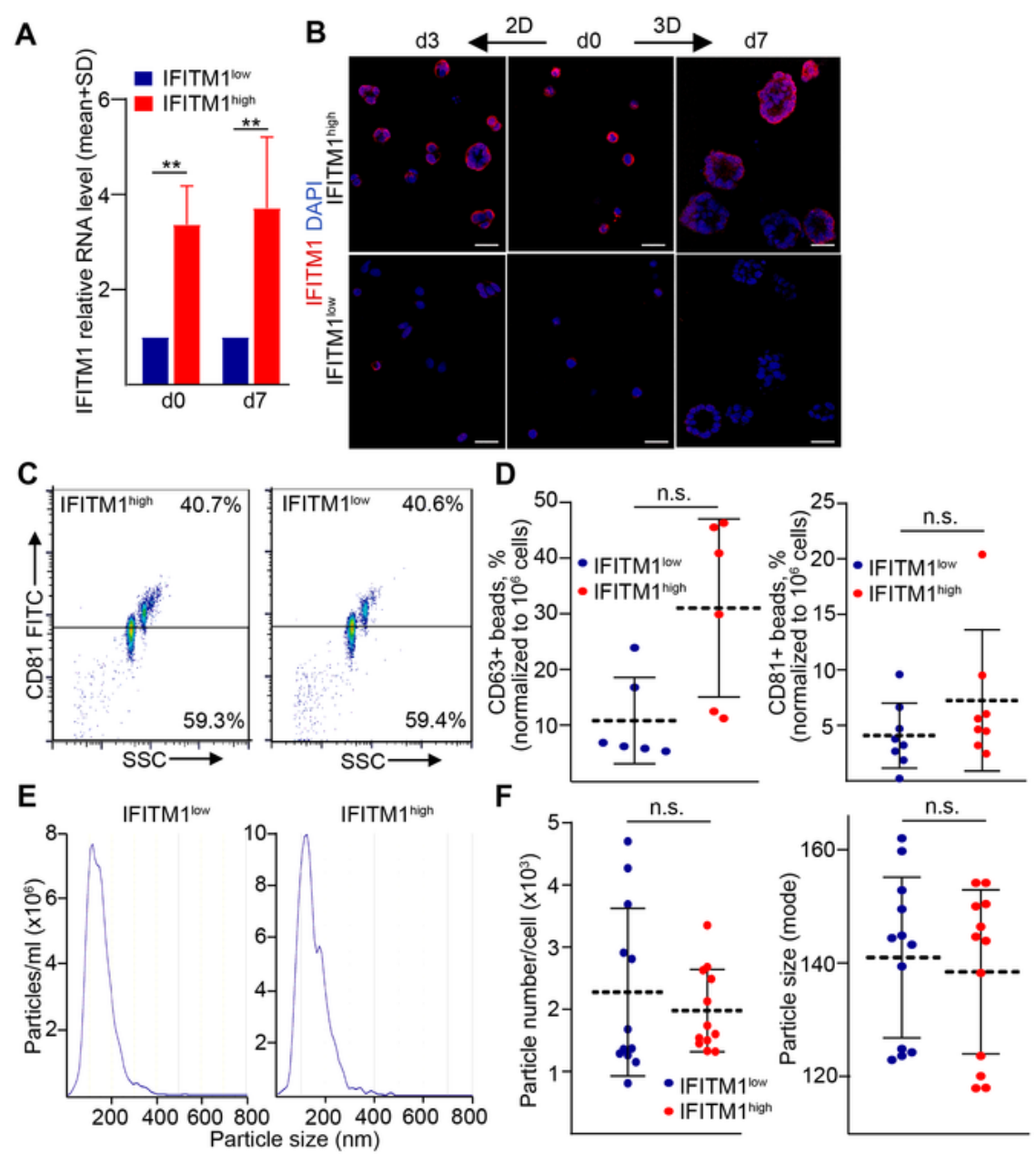

Kelemen, Figure 3.

Figure 3 
The IFITM1 high and IFITM1low CRC cell-derived organoids do not differ in their EV release. A) Relative IFITM1 RNA level of sorted cells (d0) and organoids derived from the sorted cells (d7). The RNA levels (normalized to housekeeping) of IFITM1low samples were taken as 1 in each experiment (RT-qPCR, $n=4$ ). B) Immunostaining for IFITM1 directly after sorting or after culturing in 2D or 3D conditions for the indicated time periods (immunostaining and whole-mount immunostaining, confocal microscope images). C-D) The percentage of anti-CD63 or anti-CD81-coated positive beads after incubating them in IFITM1 low or IFITM1 high organoid-derived supernatants and detected by anti-CD63 or anti-CD81 antibody, respectively (flow cytometry). Representative plots (C) and quantification of the data (D). Note that data were normalized to cell number. E-F) Nanoparticle Tracking Analysis (NTA) of the organoid supernatants after ultracentrifugation. Representiative NTA images (E) and data normalized to cell number (F). Scale bars: $50 \mu \mathrm{m}(B)$. Paired t-test (A) and Mann-Whitney $U$ tests $(D, F)$ were used with $* * p<0.01$ and n.s.: $p>0.05$. 

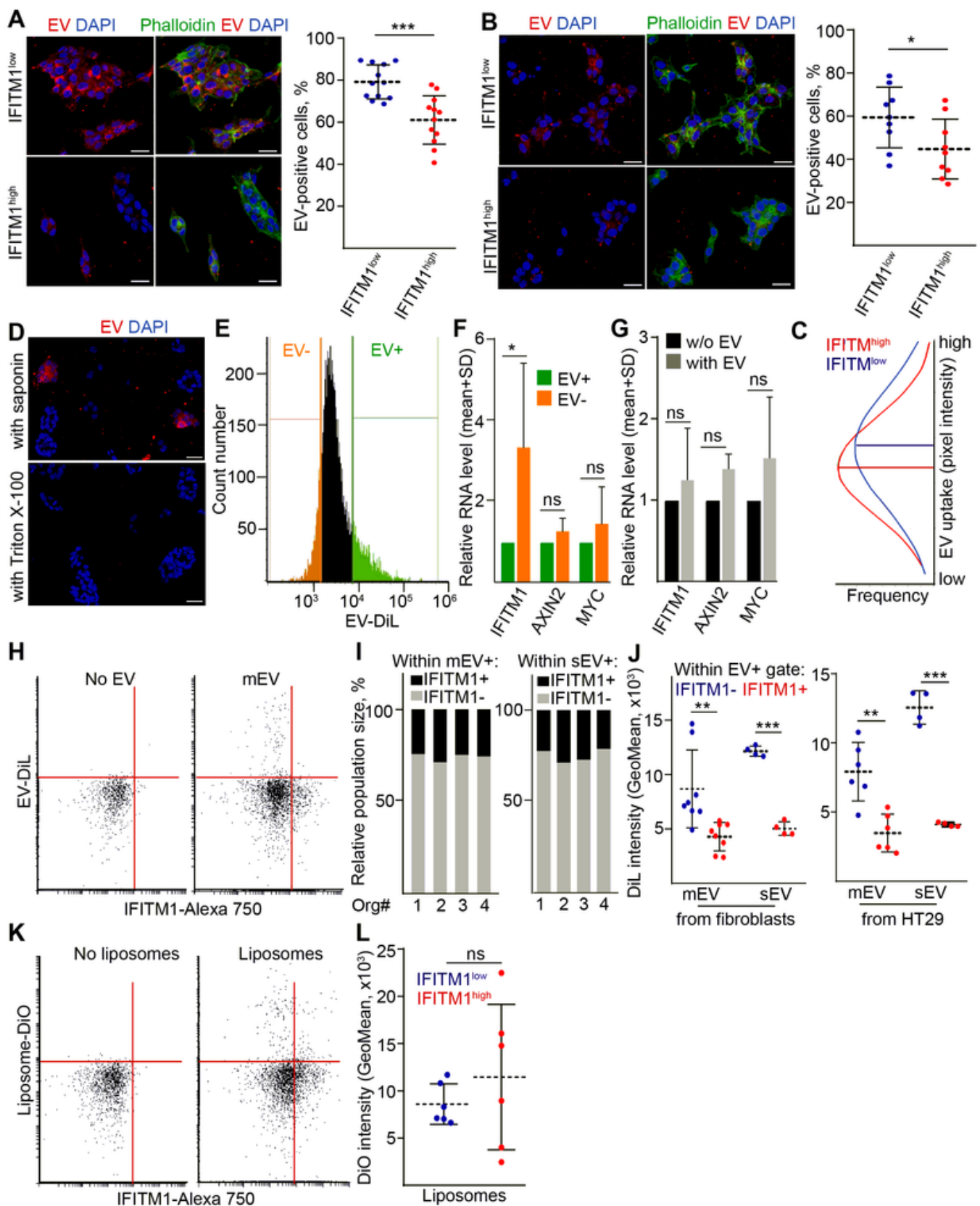

Kelemen, Figure 4.

\section{Figure 4}

IFITM1high cells have a diminished EV uptake ability. A-B) Medium EV (mEV) uptake in IFITM1 low and IFITM1high sorted cells. Representative images (left panels) and their quantification (right panels). Sorted cells were cultured 2D for 3 days and they were treated with mEVs derived from DiL-labelled HT29 CRC cells $(A)$ or human colon fibroblasts $(B)$. Note that $\mathrm{mEVs}$ show red fluorescence and phalloidin was used to visualize cells. Confocal microscope images were taken from two independent experiments. The 
shown images are optical slices from the inside of the cells. C) Distribution of the DiL signal intensity in IFITM1low and IFITM1high cells. Cells were treated with mEVs derived from DiL-labelled fibroblasts. Note the shift of the curve between the two cell populations. D) The fluorescent red signal of mEVs in IFITM1 low sorted cells, cultured for 3 days in 2D conditions. mEVs were collected from DiL-labelled fibroblasts and samples were treated with saponin or Triton X-100. Note that the EV signal disappears when using Triton X-100. E) The sorting of CRC organoid cells pre-treated with DiL labelled fibroblastderived small EVs (sEV) overnight in 2D cultures. F) Relative RNA levels of IFITM1, AXIN2 and MYC in the sorted EV+ and EV negative CRC cells (RT-qPCR, n=3). RNA levels (normalized to housekeeping) of the $\mathrm{EV}+$ samples was always taken as 1. G) Relative RNA of the indicated genes in CRC organoid cells (cultured in 2D conditions) with or without fibroblast-derived sEVs (RT-qPCR, n=3). H) IFITM1 level and EV uptake of CRC organoid-derived cells (flow cytometry) in the presence/absence of fibroblast-derived mEVs. I) The relative percentage of IFITM1 high and IFITM1low cells within cells with mEV (left panel) or sEV (right panel) in four CRC organoid lines (flow cytometry). J) EV-DiL red fluorescent signal intensity in IFITM1-/low and IFITM1high cells after treatment with mEVs or sEVs derived from fibroblasts (left panel) or HT29 cells (right panel). Four organoid lines were measured twice (flow cytometry). K) IFITM1 level and the uptake of liposomes labelled with DiO (representative flow cytomtery images). L) DiO green fluorescent signal intensity within the DiO-liposome+ population (data were collected form three organoid lines in two experiments). Scale bars: $20 \mu \mathrm{m}(A, B, C)$. Paired t-tests (F, G), t-test (C), Mann-Whitney U-test $(A, B, J, L)$ were used with ${ }^{*} p<0.05,{ }^{* *} p<0.005$ and n.d.: $p>0.05$. 

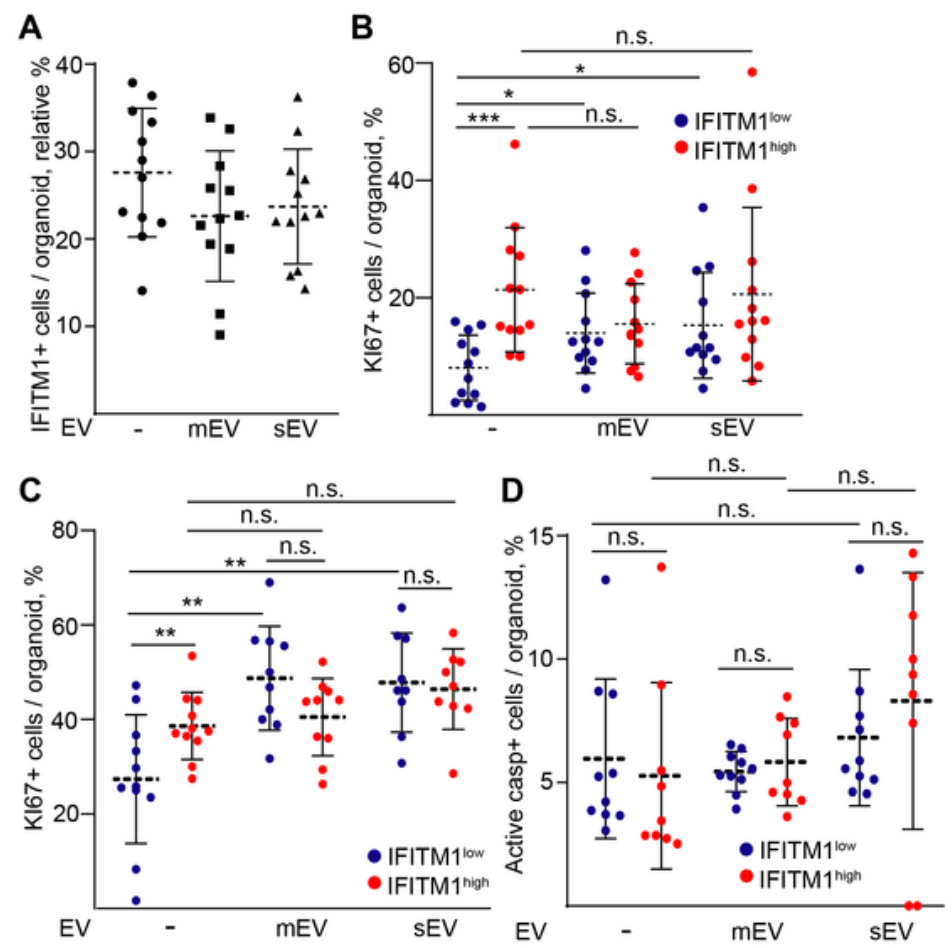

Kelemen, Figure 5 .

\section{Figure 5}

Fibroblast-derived EVs induce the proliferation of IFITM1 low CRC cells. A) The proportion of IFITM1+CRC cells in the absence of fibroblast-derived EVs or after adding sEVs or mEVs. B) The percentage of IFITM1low/KI67+ and IFITM1 high/KI67+ cells in CRC organoids after the indicated treatments. C) The percentage of KI67+ proliferating cells in IFITM1high or IFITM1 low CRC cell-derived organoids, treated with fibroblast sEVs or mEVs directly after sorting (whole-mount immunostaining and confocal 
microscope images). D) The proportion of active caspase-3+ apoptotic cells in CRC organoids derived from the indicated cell populations, cultured with or without $\mathrm{mEV}$ s or $\mathrm{sEV}$ s. Images were taken from three experiments for $A, B, C$ and $D$. Two-way ANOVA and Tukey post hoc tests were carried out $(A, B, C, D)$ with ${ }^{*} \mathrm{p}<0.01$ and n.s.: $p>0.05$. Note that only the relevant comparisons are shown.

\section{Supplementary Files}

This is a list of supplementary files associated with this preprint. Click to download.

- SupplinfFigures.pdf 